\title{
Non-genomic progesterone signalling and its non-canonical receptor
}

Patricia Moussatche and Thomas J. Lyons ${ }^{1}$

Foundation for Applied Molecular Evolution, P.0. Box 13174, Gainesville, FL 32604, U.S.A.

\begin{abstract}
The steroid hormone progesterone regulates many critical aspects of vertebrate physiology. The nuclear receptor for progesterone functions as a ligand-activated transcription factor, directly regulating gene expression. This type of signalling is referred to as the 'genomic' pathway. Nevertheless, progesterone also stimulates rapid physiological effects that are independent of transcription. This pathway, termed 'nongenomic', is mediated by the mPRs (membrane progesterone receptors). These mPRs belong to a larger class of membrane receptors called PAQRs (progestin and adipoQ receptors), which include receptors for adiponectin in vertebrates and osmotin in fungi. mPRs have been shown to activate inhibitory G-proteins, suggesting that they act as GPCRs (G-protein-coupled receptors). However, PAQRs do not resemble GPCRs with respect to topology or conserved sequence motifs. Instead, they more closely resemble proteins in the alkaline ceramidase family and they may possess enzymatic activity. In the present paper, we highlight the evidence in support of each model and what is currently known for PAQR signal transduction of this non-canonical receptor.
\end{abstract}

\section{Introduction}

Progesterone is a steroid hormone that regulates female reproduction and other critical aspects of vertebrate physiology. Studies of progesterone signalling have mostly focused on the nuclear receptor, which functions as a progesterone-activated transcription factor and directly regulates gene expression [1] This type of signalling is referred to as the 'genomic' pathway. However, progesterone also stimulates many rapid responses that are independent of transcription, such as changes in ion flux and intracellular $\mathrm{Ca}^{2+}$, along with other second messengers [2]. This 'non-genomic' pathway is mediated by the mPRs (membrane progesterone receptors). mPRs have been identified not only in male and female reproductive tissues, but also in immune cells and tissues, the nervous system and cancer cells [2-6].

$\mathrm{mPRs}$ were first identified in fish ovaries $[7,8]$, and a thorough review of the cloning and characterization of these receptors can be found in [2]. These mPRs belong to a larger class of membrane receptors called PAQRs (progestin and adipoQ receptors), which include receptors for adiponectin in vertebrates and osmotin in fungi [9]. The phylogenetic tree shown in Figure 1 demonstrates the relationship between the mPRs, PAQRs and other membrane proteins and shows that the $\mathrm{mPR}$ clade diverged from the rest of the PAQR family quite early in the evolution of metazoans,

Key words: ceramidase, G-protein, non-canonical receptor, progesterone, progestin and adipoQ receptor (PAQR), signalling.

Abbreviations used: AdipoR, adiponectin receptor; AlkCer, alkaline ceramidase; AMPK, AMPactivated protein kinase; APPL1, adaptor protein, phosphotyrosine interaction, pleckstrin homology domain and leucine zipper-containing 1; GPCR, G-protein-coupled receptor; MPR, membrane progesterone receptor; PAQR, progestin and adipoQ receptor; PTX, Pertussis toxin S1P, sphingosine 1-phosphate; TM, transmembrane domain; TNF $\alpha$, tumour necrosis factor $\alpha$. ${ }^{1}$ To whom correspondence should be addressed (email tlyons@ffame.org). with representatives being found in simple placozoans. Thus progesterone signalling via the mPRs may precede signalling via the nuclear receptor [10] and may be the oldest form of steroid signalling in animals.

The predicted PAQR hydropathy shows seven TMs (transmembrane domains), comparable with GPCRs (Gprotein-coupled receptors), and the signalling pathway they control seems to activate inhibitory G-protein cascades in female reproductive tissues [7,8,11-13]. However, this novel family of receptors lacks significant sequence similarity to any known GPCRs and may have opposite transmembrane topology. Moreover, a detailed sequence analysis of the PAQR family revealed that these receptors show sequence motifs characteristic of AlkCers (alkaline ceramidases) and present enzymatic activity as such $[14,15]$. A dispute for the true second messenger has occupied the literature in recent years and we highlight the evidence in support of each pathway.

\section{Evidence for G-protein signalling}

Even before the first $\mathrm{mPR}$ was cloned [7,8], it was suggested that progestin-dependent maturation of rainbow trout (Oncorbynchus mykiss) oocytes occurred via inhibitory G-proteins $\left(G_{i}\right)$ [16]. It was observed that progestin treatment inhibited adenylate cyclase and lowered cAMP levels. Moreover, progestin increases non-hydrolysable GTP binding to membranes, whereas progestin binding decreases in the presence of non-hydrolysable GTP. PTX (Pertussis toxin), which prevents $G_{i}$ from interacting with its receptor by preventing the exchange of bound GDP, was used to determine the identity of the G-protein involved. The same 
Figure 1 Bootstrapped phylogenetic tree of PAQRs, AlkCers and GPCRs

The tree was generated by ClustalX using the neighbour-joining method with protein datasets truncated to include only the seven-TM core regions. Bootstrap values at the nodes are confidence values indicating the number of times per 1000 trees a particular grouping is made. Branch lengths are proportional evolutionary distance and the scale bar indicates the number of substitutions per site. Note: The AlkCers form a sister group to the PAQRs, whereas the human Class A GPCRs are highly divergent. The high bootstrap value at the node separating the human Class A GPCRs from PAQRs and AlkCers indicates a high degree of confidence in the inclusion of PAQRs and AlkCers into the same clade. However, the long branch lengths in the GPCR clade indicate that little unifies this family in terms of conserved sequence motifs. mPRs clearly form a group within the PAQR clade that we have termed Class II. The sister group to the mPRs is the clade containing the AdipoRs (Class I). Also, it is clear from this analysis that the simple placozoan Trichoplax adhoerens contains Class I, Class II and Class III PAQRs, suggesting the mPRs arose early in metazoan evolution. OsmoR, osmotin receptor.

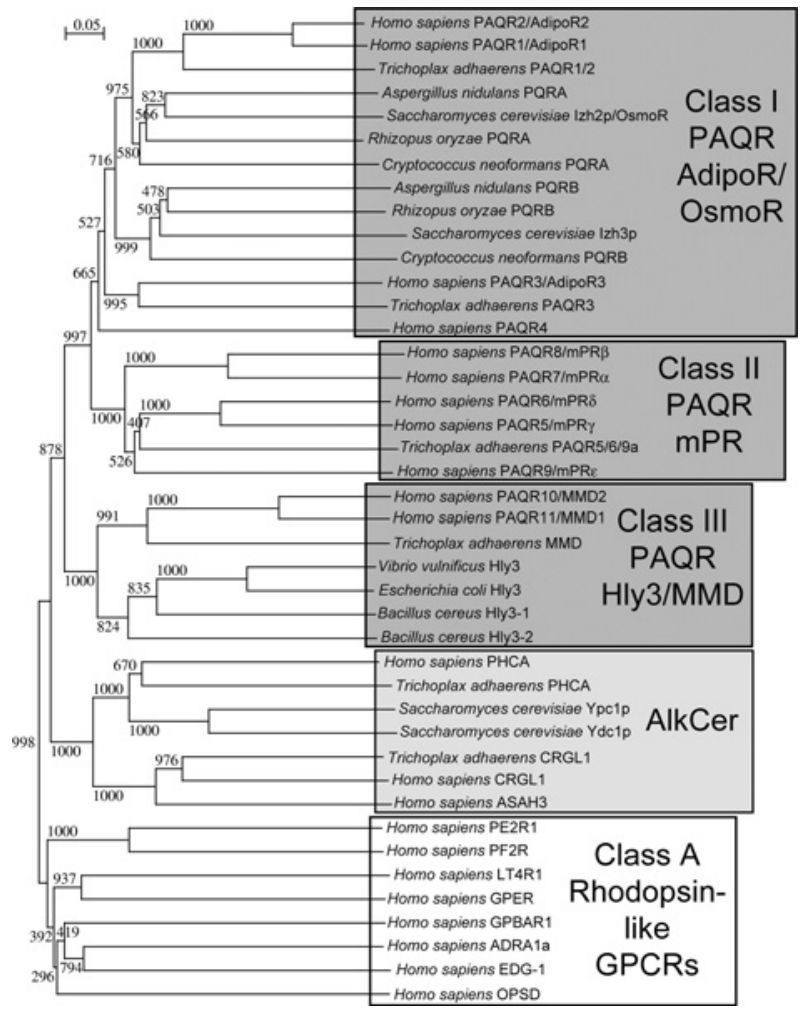

was shown for Atlantic croaker (Micropogonias undulatus) oocytes [5], human myometrium [4], immortalized T-cells and leucocytes [3], and mouse hypothalamus and neuroblastoma cell lines [6].

Cloning of mPRs allowed expression in breast cancer cells lacking nuclear receptors, which facilitated non-genomic progestin signalling studies $[7,8]$. Stable transfections of fish and human mPRs in breast cancer cell lines displayed the same responses to non-hydrolysable GTP and PTX [13]. Moreover, the $\mathrm{mPR} \alpha$ subtype could be detected in immunoprecipitated $G_{i}$ pools, and this coupling was lost after progestin treatment. Co-immunoprecipitation of $G_{i}$ and mPRs also has been shown in human myometrium [4]. However, transfections with two different $\mathrm{mPR}$ isoforms from zebrafish (Danio rerio) showed that both $\mathrm{mPR} \alpha$ and $\operatorname{mPR} \beta$ could decrease cAMP levels after progestin stimulation in a dose- and time-dependent manner, whereas only $\mathrm{mPR} \alpha$ was affected by PTX [17].

Most of the G-proteins associated with progestin nongenomic signalling to date have been of the inhibitory type. An exception worth noting is the activation of olfactory G-protein in Atlantic croaker sperm [18]. Progestin treatment increases cAMP concentrations in this system, and stimulation is prevented by Cholera toxin, which is an activator of stimulatory G-proteins as it prevents bound GTP from hydrolysing. Progestin treatment increases non-hydrolysable GTP binding to croaker membranes, and only olfactory G-proteins were detected, which coimmunoprecipitated with $\operatorname{mPR} \alpha$.

Despite the results described above, several findings have led to widespread unease with the categorization of mPRs as GPCRs. First, some groups have had difficulty confirming the activation of $G_{\alpha}$ proteins by mPRs [19]. Secondly, interactions have been inferred from co-immunoprecipitation assays of intact cellular membranes, which are likely to contain a heterogeneous mixture of membrane proteins. Thirdly, human mPRs can be functionally expressed in Saccharomyces cerevisiae, which does not express human $\mathrm{G}_{\alpha}$, and even functionally respond to progesterone in yeast cells lacking any endogenous $\mathrm{G}_{\alpha}$ [10]. This suggests that G-proteins are not an essential component of PAQRmediated signalling. That said, some studies suggest that Gproteins are important components of endogenous PAQR signalling in fungi $[20,21]$, so the issue is not entirely resolved.

The only physical resemblance between GPCRs and PAQRs is the presence of a seven-TM core. In fact, phylogenetic analysis by Smith et al. [10] showed no apparent relationship between GPCRs and PAQRs. Rather, the phylogenetic tree in Figure 1 suggests that PAQRs are more similar to other seven-TM-containing protein families than they are to GPCRs. To complicate matters, the topology of this core in PAQRs is $\mathrm{N}_{\text {in }}, \mathrm{C}_{\text {out }}$ [22-24], rather than the characteristic $\mathrm{N}_{\text {out }}, \mathrm{C}_{\text {in }}$ topology of GPCRs. A model for the topology of PAQRs is shown in Figure 2. To be fair, not all GPCRs seem to conform to this characteristic topology [25], and it is even possible for some proteins to adopt multiple topologies [26]. Moreover, conflicting results have been published for the topology of proteins in the mPR subgroup, which possess an eighth TM C-terminal to the seven-TM PAQR core $[10,13]$. PAQRs and GPCRs do not share any conserved sequence motifs, but there are no conserved motifs that unify the entire GPCR family, a fact that has led some to speculate that the GPCR 'superfamily' is actually a polyphyletic group whose divergent members arrived at similar functions via homoplasy [27]. Ultimately, a canonical GPCR is defined as a receptor that sequesters transducer proteins (e.g. heterotrimeric G-proteins) at the membrane and releases them as second messengers upon binding of 
Figure 2 | Topological model for PAQRs and AlkCers

A topology model for the PAQR family showing the seven-TM core, the $\mathrm{N}_{\text {in, }} \mathrm{C}_{\text {out }}$ topology and the three conserved motifs described in the text. The AlkCer family is characterized the by a seven-TM core with the same topology and the same three conserved motifs. The MPR subgroup of the PAQRs (also referred to as Class II PAQRs) are characterized by an additional TM that is C-terminal to the seven-TM core.

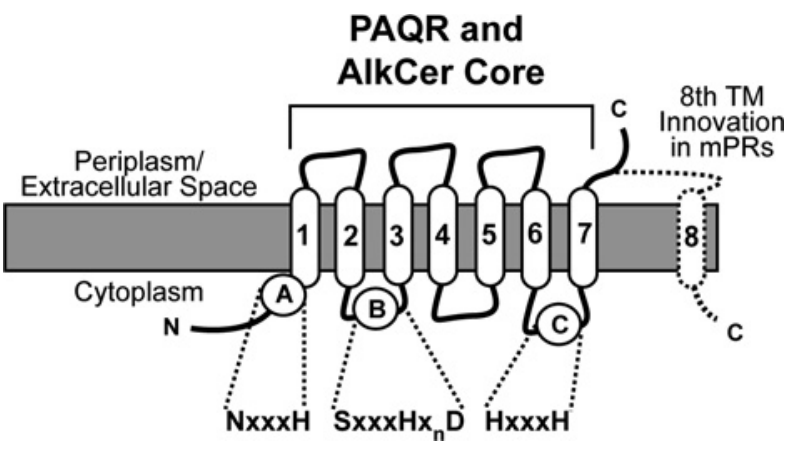

agonist. However, this definition is also troublesome, since there is evidence that some GPCRs do not absolutely require such transducers to initiate signalling [27]. In the end, there is little consensus as to what defines a GPCR other than the presence of the seven-TM core. Yet, enigmatically, not all proteins with a conserved seven-TM core are considered to be GPCRs (see the next section), complicating the unambiguous characterization of mPRs as GPCRs.

It is also important to note that G-proteins are not implicated in the signalling mechanism of AdipoRs (adiponectin receptors) [28]. The close relationship between AdipoRs and mPRs suggests that the two PAQR subfamilies may be functionally related, or at least work via similar signalling mechanisms. This is supported by the fact that both AdipoRs and mPRs can functionally couple to the same signal transduction pathway in yeast, suggesting a common mechanism of action [29]. Recently, Heiker et al. [28] succinctly reviewed what is known about the signalling pathway downstream of the AdipoRs. This involves dimerization of receptors, AMPK (AMP-activated protein kinase), APPL1 (adaptor protein, phosphotyrosine interaction, pleckstrin homology domain and leucine zipper-containing 1) and RACK1 (receptor for activated C-kinase 1). Some of these components are conserved in the signalling pathway in yeast (i.e. AMPK and APPL1) [29]. Unfortunately, the adiponectin and progesterone signalling research fields have largely proceeded in parallel without cross-talk. As the similarities between the AdipoRs and mPRs became more apparent, there is now a growing discussion of how the two fields may be interrelated $[14,30]$.

\section{Evidence for sphingolipid signalling}

The unifying feature of the entire PAQR family is the presence of three highly conserved motifs at precise locations in the seven-TM core (Figure 2). Motif A (TM1) has the consensus NXXXH, Motif B (TM2-TM3) has the consensus $\mathrm{SXXXHX}_{n} \mathrm{D}$ and Motif C (TM6-TM7) has the consensus $\mathrm{HXXXH}[10,15,31]$. Intriguingly, these same three motifs can be found in exactly the same positions in another protein family that is characterized by a seven-TM core: the AlkCer [15] (also in Figure 2). In addition, the AlkCer family appears to have the same topology as the PAQR family [24]. AlkCers catalyse the reversible deacylation of ceramides to produce sphingoid bases. Both ceramides and sphingoid bases function as potent lipid second messengers in eukaryotic cells. The ratio of ceramides to sphingoid bases is often referred to as the ceramide rheostat, which globally regulates cellular metabolism [32]. The sequence similarity between the PAQRs and AlkCers suggests that the PAQRs might possess ceramidase activity. Indeed, a role for ceramides and sphingoid bases in PAQR signalling was first shown in yeast [15] and, subsequently, in mammalian cells [33]. Furthermore, whereas the addition of sphingoid base or overexpression of a known AlkCer recapitulates the effect of $\mathrm{mPRs}$ in the heterologous yeast expression system, the AlkCer inhibitor D-erythro-MAPP [(1S,2R)-D-erythro2-(N-myristoylamino)-1-phenylpropan-1-ol] inhibits $\mathrm{mPR}$ signalling [14]. On the basis of these results and the similarities between the two families, it has been proposed that PAQRs might function as ligand-regulated AlkCer enzymes [15].

Although the ceramidase model for signal transduction via PAQRs was only recently proposed by Villa et al. [15], a role for sphingolipids in non-genomic progesterone signalling had already been established [34-37]. Indeed, in the oocyte maturation model of non-genomic progesterone signalling, the addition of sphingoid base can replace progesterone as the inducer of germinal vesicle breakdown [37]. Intriguingly, the AlkCer family of enzymes seems to catalyse both ceramidase and ceramide synthase reactions [38], and it is possible that the PAQRs might possess both activities as well. It has been proposed that PAQRs might be in the unique position to control many aspects of cellular metabolism as a fulcrum for the ceramide rheostat, depending upon whether or not ceramidase or ceramide synthase activity is favoured [14]. In support of this model, both agonists (progestins) and inverse agonists [TNF $\alpha$ (tumour necrosis factor $\alpha$ )] have been identified for the mPRs using the yeast system as a functional assay. TNF $\alpha$ is a homologue of adiponectin that is known to generate ceramide second messengers. It is known that TNF $\alpha$ counteracts the effects of both progesterone and adiponectin, therefore the $\mathrm{mPR}$ and AdipoR families may be unified by their antagonism to TNF $\alpha$ [14].

The established role of $G_{i}$ in progesterone signalling described above, as well as some other non-genomic effects of progesterone, could alternatively be explained as a downstream effect of sphingolipid signalling. Phosphorylated sphingoid bases [S1P (sphingosine 1-phosphate)] are known activators of receptors in the EDG subfamily of GPCRs and the S1P receptors are known to couple to $G_{i}$ [39]. Since both PAQRs and S1P receptors are involved in sphingolipid signalling, it would not be surprising if they co-localized to membrane microdomains or 'lipid rafts' that are enriched 
Figure 3 Signal transduction models

Two distinct models for the mechanism of signalling via mPRs have been proposed. In the GPCR model, the mPRs function as GPCRs that sequester inactive G-proteins and release them upon binding of progesterone. In the Alkcer model, the mPRs function as AlkCer enzymes that interconvert ceramides and sphingoid bases, which function as potent second messengers, including in the activation of the EDG-1 S1P receptor, a known GPCR. Since MPRs are distinguished from other PAQRs by the presence of an eighth $T M$ at the C-terminus, we have proposed a third 'dual' model where the seven-TM PAQR core functions as an AlkCer, whereas the C-terminus unique to mPRs adds GPCR functionality.
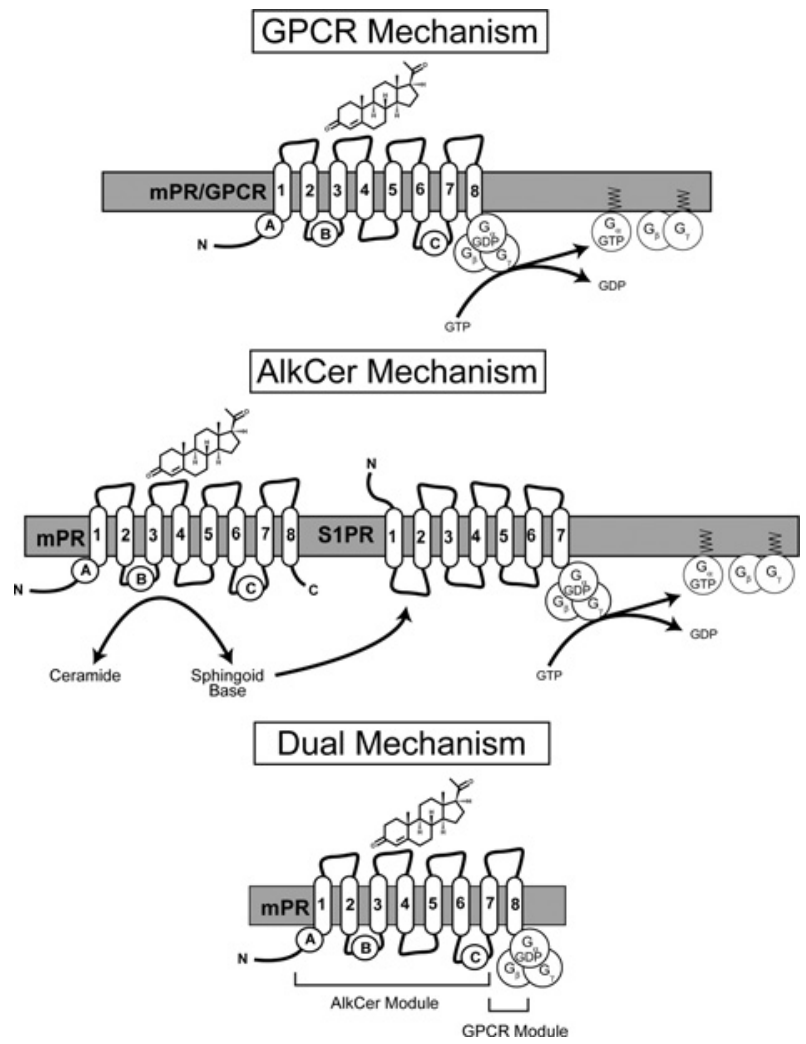

in sphingolipids. Indeed, this seems to be true for the EDG-1 S1P receptor [40] as well as for the yeast PAQR Izh2p (N. Villa and T. Lyons, unpublished work). Colocalization of mPRs and a S1P receptor to detergent-resistant microdomains might also explain co-immunoprecipitation results, suggesting an association between $\mathrm{G}_{\mathrm{i}}$ and the $\mathrm{mPR}$.

\section{The best of both worlds}

We have suggested that $G_{i}$ binding to the mPRs, and therefore the GPCR model for signalling, may be an artefact of the co-immunoprecipitation assay used. However, this need not be the case. Indeed, the GPCR and AlkCer models for PAQR signalling are not necessarily mutually exclusive (Figure 3). As mentioned above, the mPRs differ from all other PAQRs by the presence of an eighth TM Cterminal to the seven-TM PAQR core. The function of this additional module is unknown, and the fact that mPRs respond normally to progesterone in the functional yeast assay system even without this domain suggests that it is not involved in progesterone binding [10]. However, Thomas et al. [13] did find that this additional module is important for G-protein binding to membranes. Therefore this module, which is unique to the mPRs, may add GPCR functionality on top of AlkCer activity by providing a docking site for heterotrimeric G-proteins, allowing dual sphingolipid/G-protein signal transduction pathways in response to progesterone (Figure 3 ). That the module is not required for progesterone-dependent signalling in the functional yeast assay system may be explained if the yeast assay only measures output from the PAQRs to the sphingolipid branch of the downstream pathway, leaving the G-protein branch unmonitored. It is possible that the published work in yeast tracks only the sphingolipid aspect of the signal transduction pathway, which is common to all PAQRs, whereas the published work in vertebrate cells has been tracking the G-protein component that is unique to mPRs. It is also possible that, like some phospholipases [41], the PAQRs function as downstream effectors of G-protein signalling whose activity is regulated by G-protein binding.

\section{Funding}

Our work is funded by the National Institutes of Health [grant numbers R01 GM086617-01 and R21 DK074812 (to T.J.L.)].

\section{References}

1 Ellmann, S., Sticht, H., Thiel, F., Beckmann, M.W., Strick, R. and Strissel, P.L. (2009) Estrogen and progesterone receptors: from molecular structures to clinical targets. Cell. Mol. Life Sci. 66, 2405-2426

2 Dressing, G.E., Goldberg, J.E., Charles, N.J., Schwertfeger, K.L. and Lange, C.A. (2011) Membrane progesterone receptor expression in mammalian tissues: a review of regulation and physiological implications. Steroids 76, 11-17

3 Dosiou, C., Hamilton, A.E., Pang, Y., Overgaard, M.T., Tulac, S., Dong, J., Thomas, P. and Giudice, L.C. (2008) Expression of membrane progesterone receptors on human T lymphocytes and Jurkat cells and activation of G-proteins by progesterone. J. Endocrinol. 196, 67-77

4 Karteris, E., Zervou, S., Pang, Y., Dong, J., Hillhouse, E.W., Randeva, H.S. and Thomas, P. (2006) Progesterone signaling in human myometrium through two novel membrane $G$ protein-coupled receptors: potential role in functional progesterone withdrawal at term. Mol. Endocrinol. 20 1519-1534

5 Pace, M.C. and Thomas, P. (2005) Steroid-induced oocyte maturation in Atlantic croaker (Micropogonios undulatus) is dependent on activation of the phosphatidylinositol 3-kinase/Akt signal transduction pathway. Biol. Reprod. 73, 988-996

6 Sleiter, N., Pang, Y., Park, C., Horton, T.H., Dong, J., Thomas, P. and Levine, J.E. (2009) Progesterone receptor A (PRA) and PRB-independent effects of progesterone on gonadotropin-releasing hormone release. Endocrinology 150, 3833-3844

7 Zhu, Y., Bond, J. and Thomas, P. (2003) Identification, classification, and partial characterization of genes in humans and other vertebrates homologous to a fish membrane progestin receptor. Proc. Natl. Acad. Sci. U.S.A. 100, 2237-2242

8 Zhu, Y., Rice, C.D., Pang, Y., Pace, M. and Thomas, P. (2003) Cloning, expression, and characterization of a membrane progestin receptor and evidence it is an intermediary in meiotic maturation of fish oocytes. Proc. Natl. Acad. Sci. U.S.A. 100, 2231-2236 
9 Tang, Y.T., Hu, T., Arterburn, M., Boyle, B., Bright, J.M., Emtage, P.C. and Funk, W.D. (2005) PAQR proteins: a novel membrane receptor family defined by an ancient 7-transmembrane pass motif. J. Mol. Evol. 61, 372-380

10 Smith, J.L., Kupchak, B.R., Garitaonandia, I., Hoang, L.K., Maina, A.S., Regalla, L.M. and Lyons, T.J. (2008) Heterologous expression of human $\mathrm{mPR} \alpha, \mathrm{mPR} \beta$ and $\mathrm{mPR} \gamma$ in yeast confirms their ability to function as membrane progesterone receptors. Steroids $\mathbf{7 3}$ $1160-1173$

11 Thomas, P. (2008) Characteristics of membrane progestin receptor $\alpha$ $(\mathrm{mPR} \alpha)$ and progesterone membrane receptor component 1 (PGMRC1) and their roles in mediating rapid progestin actions. Front. Neuroendocrinol. 29, 292-312

12 Thomas, P., Dressing, G., Pang, Y., Berg, H., Tubbs, C., Benninghoff, A. and Doughty, K. (2006) Progestin, estrogen and androgen G-protein coupled receptors in fish gonads. Steroids 71, 310-316

13 Thomas, P., Pang, Y., Dong, J., Groenen, P., Kelder, J., de Vlieg, J., Zhu, Y. and Tubbs, C. (2007) Steroid and G protein binding characteristics of the seatrout and human progestin membrane receptor $\alpha$ subtypes and their evolutionary origins. Endocrinology 148, 705-718

14 Kupchak, B.R., Garitaonandia, I., Villa, N.Y., Smith, J.L. and Lyons, T.J. (2009) Antagonism of human adiponectin receptors and their membrane progesterone receptor paralogs by TNF $\alpha$ and a ceramidase inhibitor. Biochemistry 48, 5504-5506

15 Villa, N.Y., Kupchak, B.R., Garitaonandia, I., Smith, J.L., Alonso, E., Alford, C., Cowart, L.A., Hannun, Y.A. and Lyons, T.J. (2009) Sphingolipids function as downstream effectors of a fungal PAQR. Mol. Pharmacol. 75 866-875

16 Yoshikuni, M. and Nagahama, Y. (1994) Involvement of an inhibitory $\mathrm{G}$-protein in the signal transduction pathway of maturation-inducing hormone (17 $\alpha, 20 \beta$-dihydroxy-4-pregnen-3-one) action in rainbow trout (Oncorhynchus mykiss) oocytes. Dev. Biol. 166, 615-22

17 Hanna, R., Pang, Y., Thomas, P. and Zhu, Y. (2006) Cell-surface expression, progestin binding, and rapid nongenomic signaling of zebrafish membrane progestin receptors $\alpha$ and $\beta$ in transfected cells. I. Endocrinol. 190, 247-260

18 Tubbs, C. and Thomas, P. (2009) Progestin signaling through an olfactory $\mathrm{G}$ protein and membrane progestin receptor $\alpha$ in Atlantic croaker sperm: potential role in induction of sperm hypermotility. Endocrinology $\mathbf{1 5 0}$ 473-484

19 Krietsch, T., Fernandes, M.S., Kero, J., Losel, R., Heyens, M., Lam, E.W., Huhtaniemi, I., Brosens, J.J. and Gellersen, B. (2006) Human homologs of the putative $\mathrm{G}$ protein-coupled membrane progestin receptors (mPR $\alpha$, $\beta$, and $\gamma$ ) localize to the endoplasmic reticulum and are not activated by progesterone. Mol. Endocrinol. 20, 3146-3164

20 Coca, M.A., Damsz, B., Yun, D.J., Hasegawa, P.M., Bressan, R.A. and Narasimhan, M.L. (2000) Heterotrimeric G-proteins of a filamentous fungus regulate cell wall composition and susceptibility to a plant PR-5 protein. Plant J. 22, 61-69

21 Yun, D.J., Ibeas, J.I., Lee, H., Coca, M.A., Narasimhan, M.L., Uesono, Y., Hasegawa, P.M., Pardo, J.M. and Bressan, R.A. (1998) Osmotin, a plant antifungal protein, subverts signal transduction to enhance fungal cell susceptibility. Mol. Cell 1, 807-817

22 Daley, D.0., Rapp, M., Granseth, E., Melen, K., Drew, D. and von Heijne, G. (2005) Global topology analysis of the Escherichio coli inner membrane proteome. Science 308, 1321-1323

23 Deckert, C.M., Heiker, J.T. and Beck-Sickinger, A.G. (2006) Localization of novel adiponectin receptor constructs. J. Recept. Signal Transduction Res. 26, 647-657
24 Kim, H., Melen, K., Osterberg, M. and von Heijne, G. (2006) A global topology map of the Socchoromyces cerevisioe membrane proteome. Proc. Natl. Acad. Sci. U.S.A. 103, 11142-11147

25 Lundin, C., Kall, L., Kreher, S.A., Kapp, K., Sonnhammer, E.L., Carlson, J.R., von Heijne, G. and Nilsson, I. (2007) Membrane topology of the Drosophilo OR83b odorant receptor. FEBS Lett. 581, 5601-5604

26 Rapp, M., Granseth, E., Seppala, S. and von Heijne, G. (2006) Identification and evolution of dual-topology membrane proteins. Nat. Struct. Mol. Biol. 13, 112-116

27 Alvarez, C.E. (2008) On the origins of arrestin and rhodopsin. BMC Evol Biol. 8, 222

28 Heiker, J.T., Kosel, D. and Beck-Sickinger, A.G. (2010) Molecular mechanisms of signal transduction via adiponectin and adiponectin receptors. Biol. Chem. 391, 1005-1018

29 Kupchak, B.R., Garitaonandia, I., Villa, N.Y., Mullen, M.B., Weaver, M.G., Regalla, L.M., Kendall, E.A. and Lyons, T.J. (2007) Probing the mechanism of FET3 repression by Izh2p overexpression. Biochim. Biophys. Acta 1773, 1124-1132

30 Michalakis, K.G. and Segars, J.H. (2010) The role of adiponectin in reproduction: from polycystic ovary syndrome to assisted reproduction. Fertil. Steril. 94, 1949-1957

31 Lyons, T.J., Villa, N.Y., Regalla, L.M., Kupchak, B.R., Vagstad, A. and Eide, D.J. (2004) Metalloregulation of yeast membrane steroid receptor homologs. Proc. Natl. Acad. Sci. U.S.A. 101, 5506-5511

32 Mandala, S.M., Thornton, R., Tu, Z., Kurtz, M.B., Nickels, J., Broach, J., Menzeleev, R. and Spiegel, S. (1998) Sphingoid base 1-phosphate phosphatase: a key regulator of sphingolipid metabolism and stress response. Proc. Natl. Acad. Sci. U.S.A. 95, 150-155

33 Holland, W.L., Miller, R.A., Wang, Z.V., Sun, K., Barth, B.M., Bui, H.H., Davis, K.E., Bikman, B.T., Halberg, N., Rutkowski, J.M. et al. (2011) Receptor-mediated activation of ceramidase activity initiates the pleiotropic actions of adiponectin. Nat. Med. 17, 55-63

34 Ghosh, S., Strum, J.C. and Bell, R.M. (1997) Lipid biochemistry: functions of glycerolipids and sphingolipids in cellular signaling. FASEB J. 11, 45-50

35 Morrill, G.A. and Kostellow, A.B. (1998) Progesterone release of lipid second messengers at the amphibian oocyte plasma membrane: role of ceramide in initiating the $\mathrm{G}_{2} / \mathrm{M}$ transition. Biochem. Biophys. Res. Commun. $246,359-363$

36 Morrill, G.A. and Kostellow, A.B. (1999) Progesterone induces meiotic division in the amphibian oocyte by releasing lipid second messengers from the plasma membrane. Steroids $\mathbf{6 4}, 157-167$

37 Strum, J.C., Swenson, K.I., Turner, J.E. and Bell, R.M. (1995) Ceramide triggers meiotic cell cycle progression in Xenopus oocytes: a potential mediator of progesterone-induced maturation. J. Biol. Chem. 270, 13541-13547

38 Mao, C., Xu, R., Bielawska, A. and Obeid, L.M. (2000) Cloning of an alkaline ceramidase from Saccharomyces cerevisioe: an enzyme with reverse (CoA-independent) ceramide synthase activity. J. Biol. Chem. 275, 6876-6884

39 Sanchez, T. and Hla, T. (2004) Structural and functional characteristics of S1P receptors. J. Cell. Biochem. 92, 913-922

40 Kohno, T., Wada, A. and Igarashi, Y. (2002) N-glycans of sphingosine 1-phosphate receptor Edg-1 regulate ligand-induced receptor internalization. FASEB I. 16, 983-992

41 Ross, E.M. (2011) G $\alpha_{q}$ and phospholipase C- $\beta$ : turn on, turn off, and do it fast. Sci. Signaling $\mathbf{4}$, pe 5

Received 14 July 2011

doi:10.1042/BST20110638 